\title{
Three-dimensional garment-size change modeled considering vertical proportions
}

\author{
Jun Zhang ${ }^{1}$, KyoungOk Kim², Masayuki Takatera ${ }^{2}$ \\ ${ }^{1}$ Interdisciplinary Graduate School of Science and Technology, Shinshu University, Ueda, Japan.) \\ ${ }^{2}$ Division of Kansei and Fashion Engineering, Institute for Fiber Engineering (IFES), \\ Interdisciplinary Cluster for Cutting Edge Research (ICCER), Shinshu University, Ueda, Japan) \\ takatera@shinshu-u.ac.jp
}

\begin{abstract}
Purpose

This paper proposes a size-changing method with three-dimensional garment modeling for various body sizes considering vertical body proportions in addition to horizontal dimensions, while preserving the silhouette and ease of the original garment.

\section{Design/methodology/approach}

Cross-sectional dimensions and shapes of one dress form (the Standard Body) and jacket bodice were obtained by three-dimensional scanning. We calculated horizontal multiplication factors of the relationship between the Standard Body and jacket bodice, and vertical body proportions. A target dress form was deformed using multiplication factors and vertical body proportions to construct a garment model that fitted the dress form. The method was verified using three different dress forms. The bodices of the jackets were compared with those obtained without adjusting vertical proportions.
\end{abstract}

\section{Findings}

Employing the proposed method, jacket bodices were made and fitted on target bodies while preserving the original shape. Jackets bodices made without considering vertical proportions had many wrinkles and deformed shape and poor fit around the bust line owing to the different vertical proportions. The vertical proportion is thus an important factor in the three-dimensional garment modeling of garments of different size fitted on a body.

\section{Research limitations/implications}

The proposed method is a new size-changing or grading method for a bodice that preserves the original silhouette.

\section{Originality/value}

The proposed modeling method allows the construction of jacket bodice models with complex ease allowance for different sizes considering both horizontal and vertical body proportions. The method is applicable when making individually tailored garments or ready-to-wear garments for different targets.

\section{Keywords}

Pattern Making, Size Change, Three-dimensional Garment Model, Vertical Body Proportion, Multiplication Factor

\section{Introduction}

Grading methods are applied to make various sizes of ready-to-wear clothing for target consumers from the master size of a garment. In addition, to make a garment for different markets, 
it is necessary to consider a body representative of the target market. Even if the height is the same, the body proportions relating to girth and vertical measurements could differ.

Pattern grading is a process of proportionally increasing or decreasing the size of a master pattern according to a prescribed set of body measurements (Cooklin, 1991; Price and Zamkoff, 1996). However, when grading is employed to make a pattern of different sizes using a master pattern, the methodology depends on the garment type (Price and Zamkoff, 1996; Lim and Istook, 2012). In the grading process, the ease allowance, shape, and body proportions are important factors that affect the garment silhouette and comfort. However, garment patterns are often graded using a planar pattern and it is thus difficult to make a garment while keeping the silhouette of the master pattern, especially in the case of a garment with a curved surface (Price and Zamkoff, 1996; Bye et al., 2008).

Nowadays, a three-dimensional (3D) garment surface flattening method is applied to pattern development (Cho et al., 2005; Cho et al., 2006a; Imaoka et al., 1989; Zhang et al., 2000; Sul and Kang, 2006; Kim et al., 2010; Yang and Zhang, 2007). The cited studies used 3D body measurements, body scanning data, or a free-form or parameterized deformation method to make a garment model. Kim (2015) located landmarks and baselines automatically on the body surface to flatten the 3D body surface and obtain patterns. Wang and Huang (2011) proposed the generation of various sizes of garment patterns by flattening 3D garments created from parameterized mannequins. Hsiao and Chen (2013) and Apeagyei (2010) presented methods for the surface reconstruction of 3D dress forms based on feature curves in horizontal directions for the clothing fit. Cichocka et al. (2014) generated parametric mannequins with ease allowance by modelling the human body. These models would work well for tight-fitting garments in sport and medical applications. However, those models are not applicable to a garment with complex ease allowance for styling and silhouette. Therefore, there remain many challenges in garment modeling, such as creating a complex 3D surface with dents, fitting body shapes, and preserving the original 3D shape for different bodies (Heisey et al., 1990; Umetani et al., 2011).

To create 3D garment model with complex ease allowance, Zhang et al. (2015) proposed a 3D garment modeling method that uses garment scanning data. They made a jacket bodice, which they then scanned and scaled in the horizontal direction using multiplication factors to construct garment models for required body sizes with the same height. Using the obtained garment models, they produced patterns of the jacket bodices employing a 3D pattern making system. They succeeded in making jackets for different body sizes with the same height and vertical body proportions. However, human body figures are different in not only the horizontal direction but also the vertical direction. Japanese adult bodies are classified by height and circumference in Japanese Industrial Standards (JIS) as shown in Figure 1. In addition, standard bodies differ depending on race or nationality. It is therefore necessary to consider the change in the vertical measurements in accordance with different heights.

In this study, to make a garment model of different sizes for various body types, we improved the garment modeling method taking into account both vertical body proportions and the horizontal dimension. 


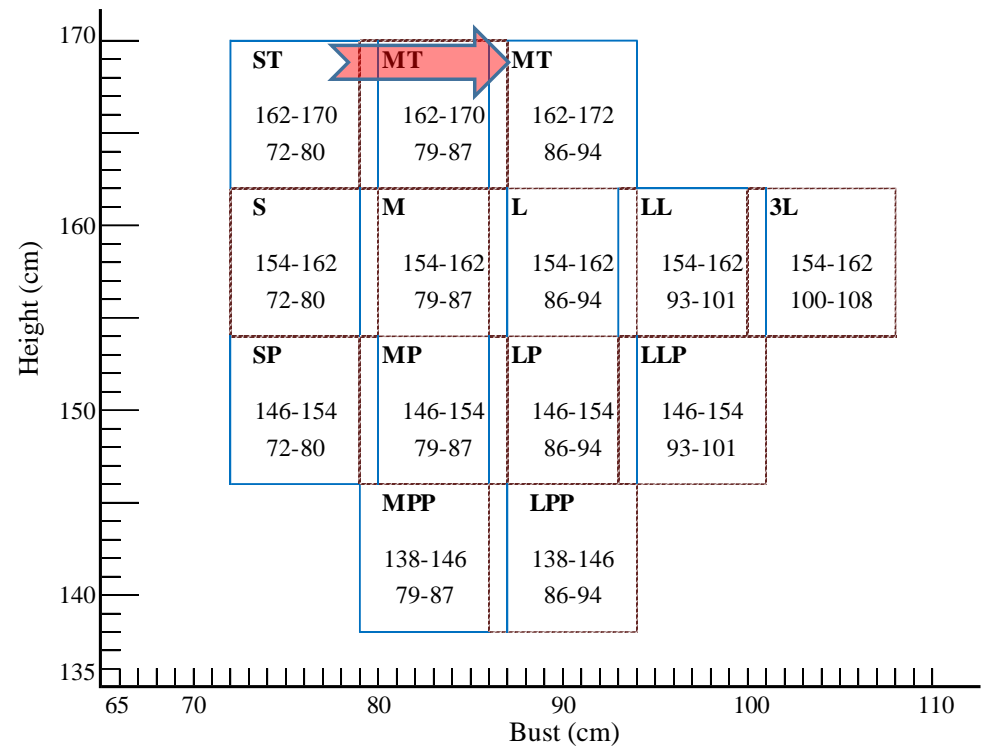

Figure 1. JIS women's body sizes. (JIS L4005)

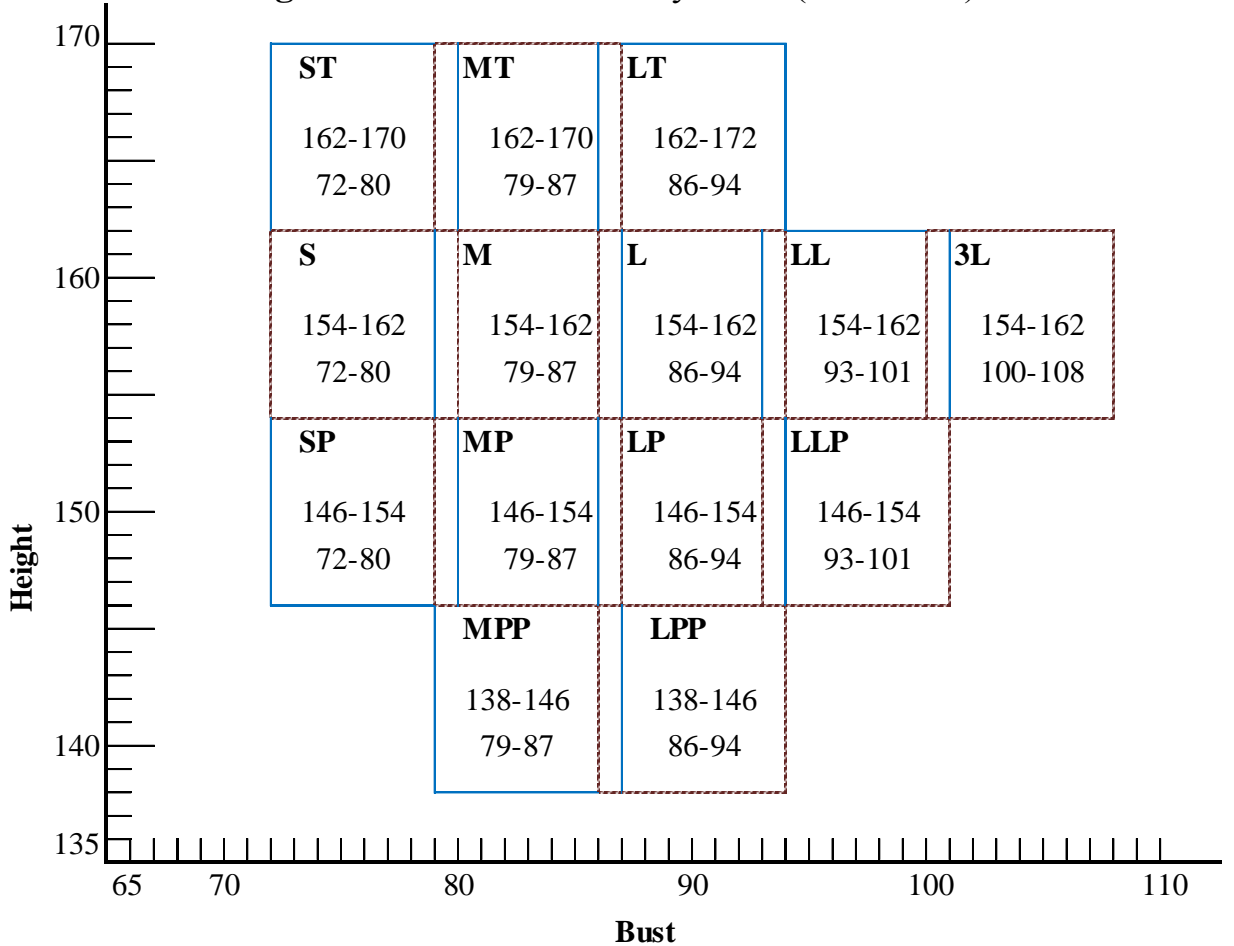

\section{Modeling Method for Different Model Sizes}

A dress form and a body-fitting jacket were selected. They are respectively referred to as the Standard Body and Standard Jacket in this paper. We obtained horizontal multiplication factors for the relationship between the cross-sections of the Standard Jacket and Standard Body (Zhang et al., 2015). Using the multiplication factors, we created another garment model that fits a target body obtained by expanding the target body horizontally and then expanding or contracting it vertically. Before expanding the body horizontally, we expanded or contracted the target body to adjust its vertical proportions to those of the Standard Body. The target body was then expanded using the multiplication factors. Afterward, the vertical proportions of the horizontally expanded 
target body were restored to the original proportions. Finally, the target body was deformed to construct the target jacket model.

\subsection{Construction of the basic model and obtaining multiplication factors for a horizontal cross-section}

3D surface data of the Standard Body and Standard Jacket worn on the body were obtained by scanning. The surface data were converted into a horizontal cross-sectional line model as shown is Figure 2 (a). A convex hull was applied to each cross-section to remove dents in the horizontal cross-section. The improved line model was converted into a polygon model, which was used as the basic garment model as shown in Figure 2 (b). Horizontal multiplication factors of the Standard Body and Standard Jacket were calculated using the distances between cross-sections in the forward, backward, and lateral directions for deformation in the horizontal direction as shown in Figure 2 (c) (Zhang et al. 2015).

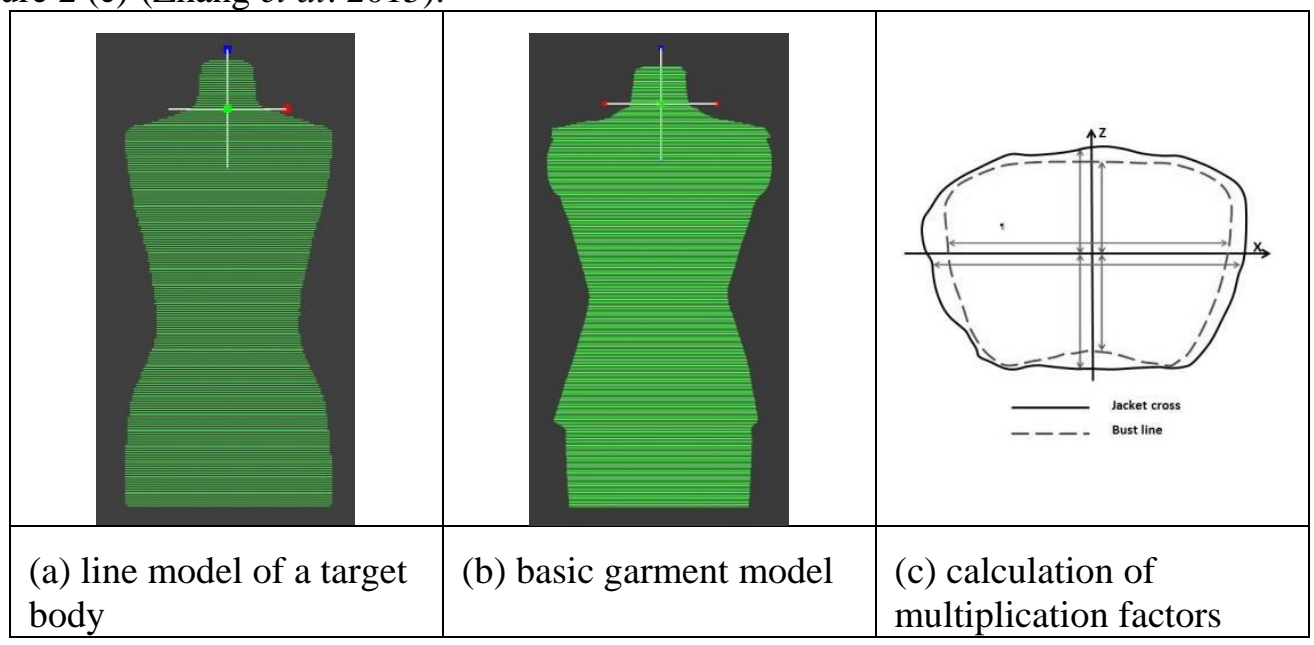

Figure 2. Scheme of modeling method for horizontal size change (Zhang et al. 2015) 2.2 Construction of garment models considering the vertical proportion

To adjust vertical proportions of the target body and Standard Body, the bodies were divided into three segments vertically, namely the front neck point (NP) to bust line (BL), the bust line to waist line (WL) and the waist line to hip line (HL) as shown in Figure 3 (a). The three vertical distances of NP-BL, BL-WL and WL-HL were measured to calculate body vertical proportions. Before applying the horizontal expansion, the vertical distances of the three segments of the target body were adjusted to those of the Standard Body using a constant multiplication factor as shown is Figure 3 (b). The target body was then deformed horizontally by applying the horizontal multiplication factors as shown is Figure 3 (c). Finally, after the vertical dimensions of the deformed body were restored to the original dimensions, we obtained a line model of the garment as shown is Figure 3 (d). The deformed line model was converted to a polygon model to develop patterns.

After constructing a 3D garment model, a 3D pattern making system (Zhang et al., 2015) was used to flatten the 3D surface to two-dimensional (2D) patterns. Covering and flattening methods were adopted to develop patterns for bodies of different sizes. 


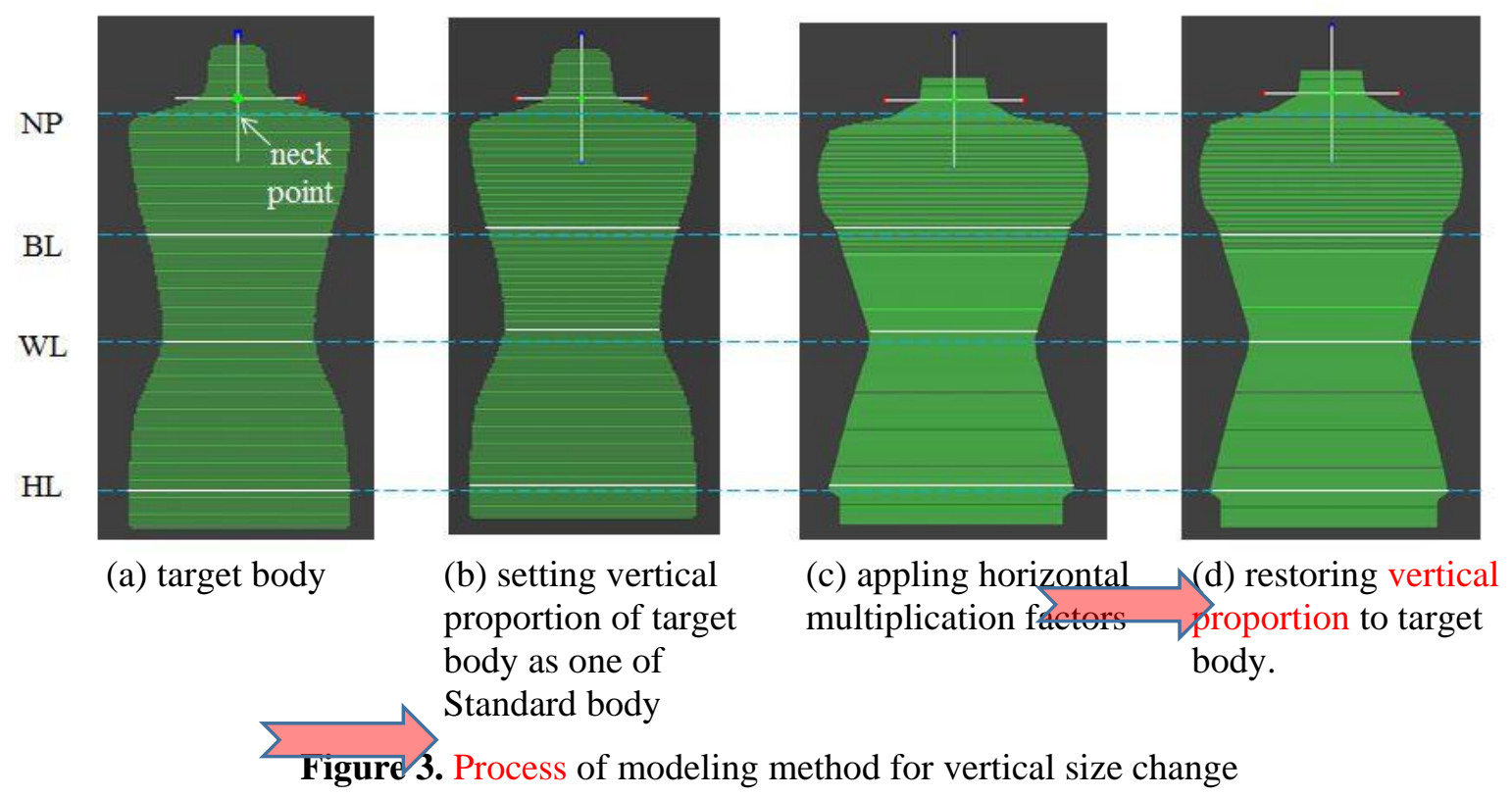

\section{Experimental}

Experiments were conducted to verify the validity of the proposed method. The Standard Jacket bodice and Standard Body were selected and scanned using a 3D scanner (Hamamatsu Photonics K.K., Shizuoka, Body Line Scanner) to obtain 3D surface data. We calculated the horizontal multiplication factors of the relationship between the Standard Body and Standard Jacket bodice.

Three target bodies with different vertical proportions were selected to construct garment models. To verify the effect of adjusting the vertical proportions, we also made garment models without adjusting the vertical proportions.

After garment models were constructed, a 3D pattern making system (Zhang et al., 2015) was used to develop patterns. The weft and warp grainlines were set at the same position as for the Standard Jacket bodice, and almost the same cutting lines as those on the garment models were set in the 3D pattern making process.

Patterns were sewn carefully to make jacket bodices with certain ease allowance. Because we focused on jacket bodice parts, all the garment models and patterns were developed without lapel, collar and sleeve parts.

Made jacket bodices were compared in terms of their silhouette, ease distribution and measurement using the 3D scanning shape data.

A Japanese female body, 9AR body (Kiiya Co., Ltd., Tokyo, Kypris 9AR) and a jacket bodice without a collar and sleeves fitted to the body were selected for the Standard Body and Standard Jacket; these are shown in Figure 4.

Three different dress forms (Body 1, Body 2 and Body 3) with different horizontal dimensions and vertical body proportions were used as target bodies in making patterns. Photographs and measurements of the dress forms are presented in Figure 5 and Table 1.

All jacket bodices were carefully made from cream-colored wool fabric (fabric weight: 149.0 $\mathrm{g} / \mathrm{m}^{2}$ ) with adhesive interlinings (fabric weight: $72.8 \mathrm{~g} / \mathrm{m}^{2}$ ) fused on each part.

Jacket names and modeling methods for target bodies are listed in Table 2. 

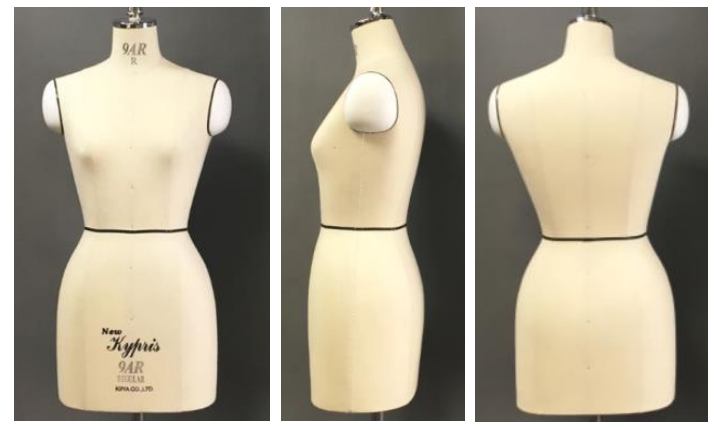

(a) Standard Body
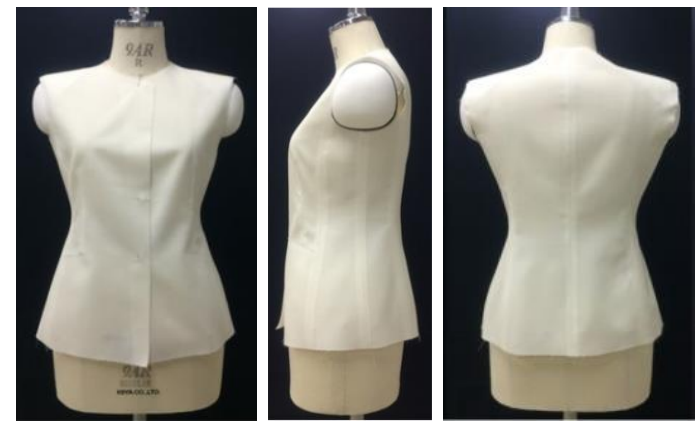

(b) Standard Jacket bodice

Figure 4. Standard Body and Standard Jacket (Zhang et al., 2015)

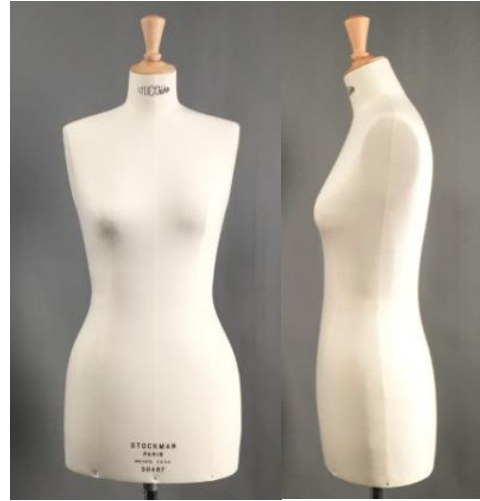

(a) Body 1

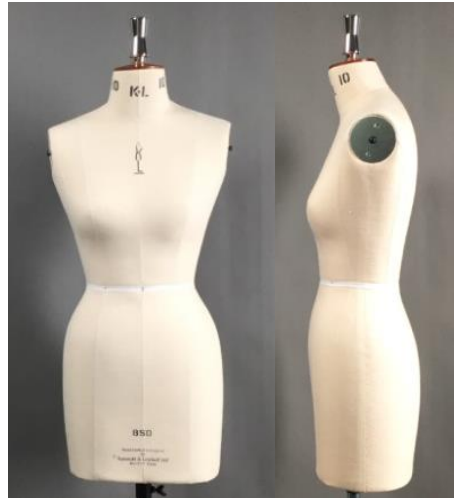

(b) Body 2

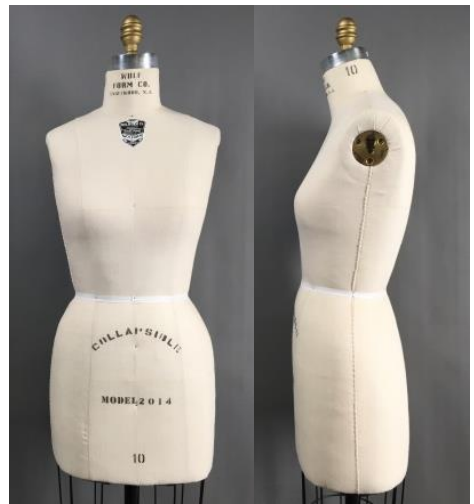

(c) Body 3

Figure 5. Photographs of three different dress forms

Table 1. Measurements of three different dress forms

\begin{tabular}{|l|c|c|c|c|c|c|c|c|}
\hline \multirow{2}{*}{ Dress form } & \multicolumn{7}{|c|}{ Measurement (cm) } \\
\cline { 2 - 9 } & BL & WL & HL & CB & SNP-BP & NP-BL & BL-WL & WL-HL \\
\hline Standard Body & 87.0 & 63.0 & 93.0 & 38.0 & 22.5 & 13.0 & 16.0 & 20.0 \\
\hline Body 1 & 87.0 & 67.0 & 94.0 & 40.5 & 24.0 & 15.0 & 16.5 & 18.0 \\
\hline Body 2 & 82.0 & 59.0 & 87.0 & 40.0 & 24.0 & 16.5 & 15.5 & 19.0 \\
\hline Body 3 & 90.2 & 66.0 & 94.0 & 43.0 & 22.5 & 17.0 & 17.7 & 18.5 \\
\hline
\end{tabular}

Annotation: $\mathrm{CB}$ denotes the surface length from the center back neck point (cervical vertebra) to the waistline, SNP-BP denotes the surface length from the side neck point to the bust point, NP$\mathrm{BL}$ denotes the vertical interval from the front neck point to the bust line, BL-WL denotes the vertical interval from the bust line to the waist line, and WL-HL denotes the vertical interval from the waist line to the hip line.

Table 2. Jacket name and modeling method for target body

\begin{tabular}{|l|c|c|}
\hline Target Body & $\begin{array}{l}\text { With adjustment of the } \\
\text { vertical proportion }\end{array}$ & $\begin{array}{l}\text { Without adjusting the } \\
\text { vertical proportion }\end{array}$ \\
\hline Body 1 & JK1 & JK1N \\
\hline Body 2 & JK2 & JK2N \\
\hline Body 3 & JK3 & JK3N \\
\hline
\end{tabular}

\section{Results and Discussion}


Three garment models were constructed taking into account both horizontal dimensions and vertical body proportions; they are shown in Figure $6(a-1),(a-2)$ and (a-3). To verify the effect of adjusting the vertical proportion, we also constructed garment models without adjusting the vertical proportions as shown in Figure 6 (b-1), (b-2) and (b-3). Developed patterns for all models are shown in Figure 7 . We made jacket bodices using these patterns. The jackets put on the dress forms are shown in Figures 8-10.
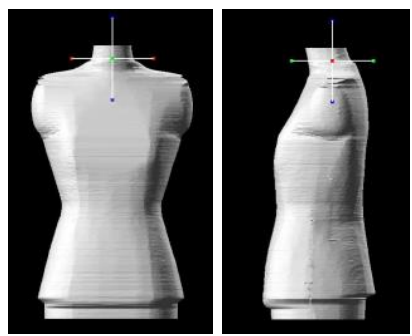

(a-1) Model for JK1
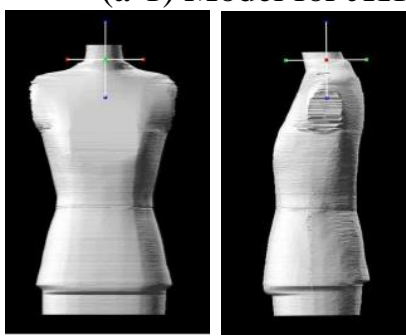

(a-2) Model for JK2
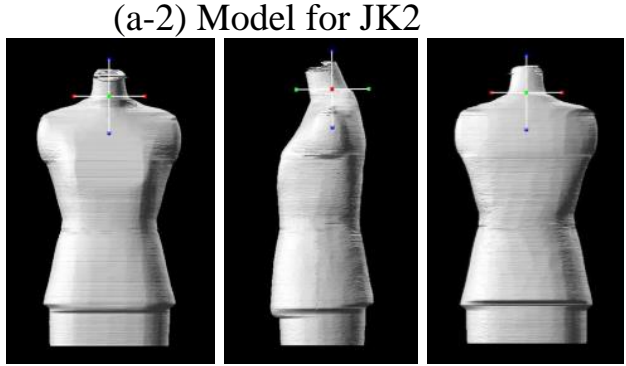

(a-3) Model for JK3
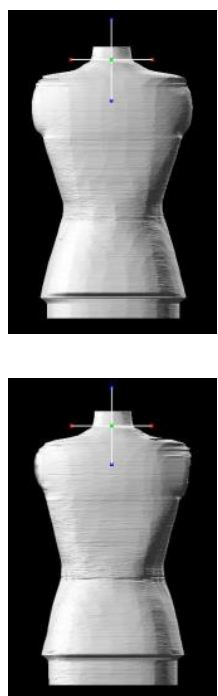

Figure 6. Jacket models obtained with and without adjusting vertical proportions
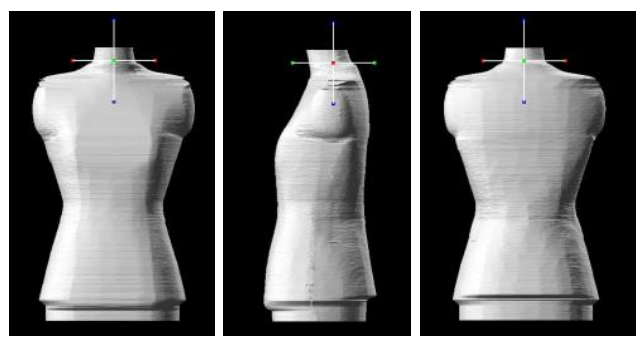

(b-1) Model for JK1N

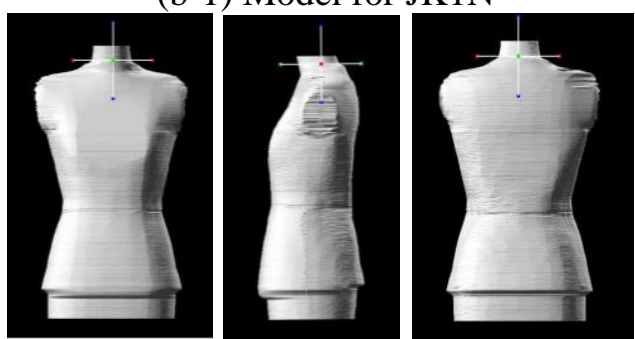

(b-2) Model for JK2N

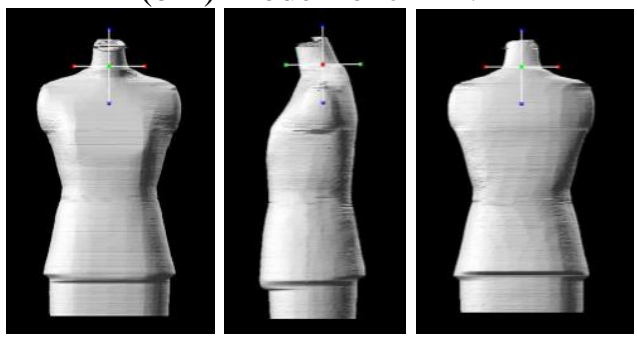

(b-3) Model for JK3N 


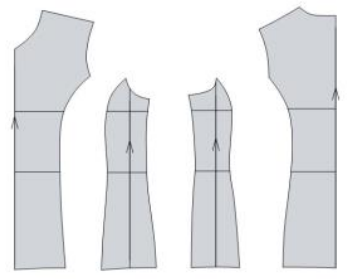

(a-1) Pattern for JK1
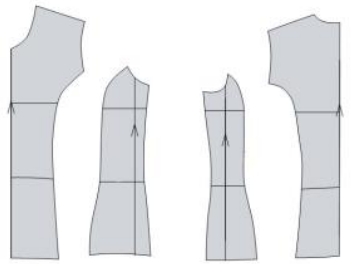

(a-2) Pattern for JK2
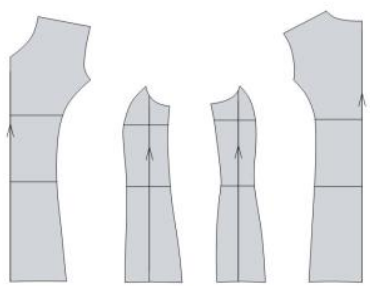

(a-3) Pattern for JK3

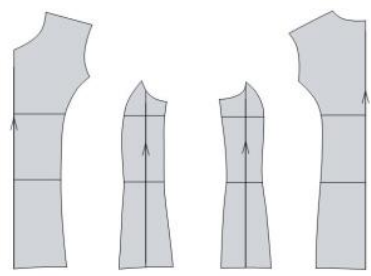

(b-1) Pattern for JK1N

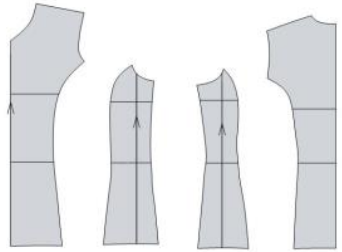

(b-2) Pattern for JK2N

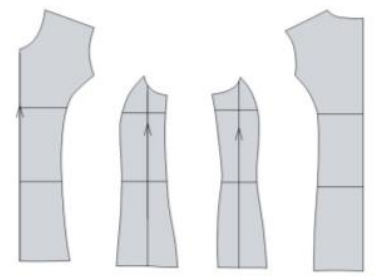

(b-3) Pattern for JK3N

Figure 7. Jacket patterns obtained with and without adjusting vertical proportions
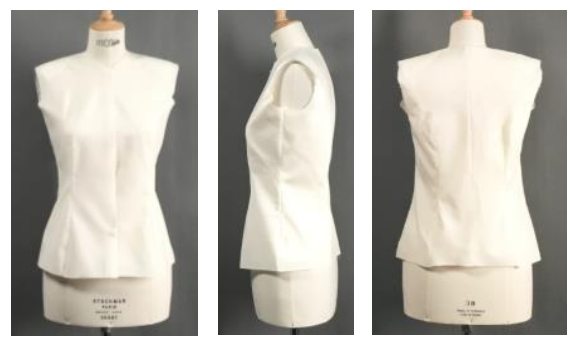

(a) JK1

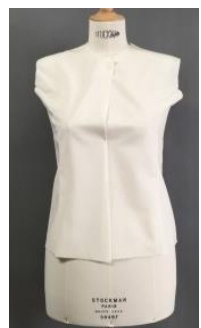

(b) JK1N

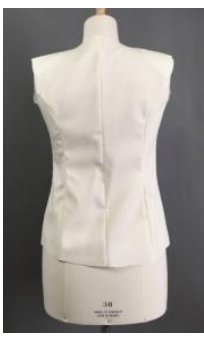

Figure 8. Jacket bodices for Body 1

(a) JK2

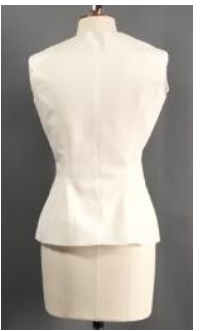

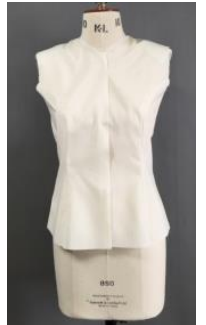

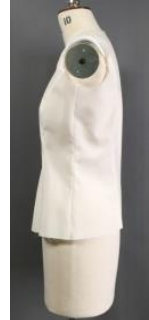

(b) JK2N

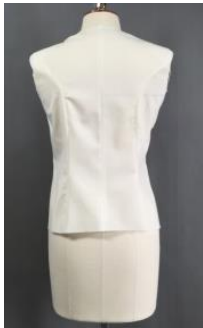

Figure 9. Jacket bodices for Body 2 


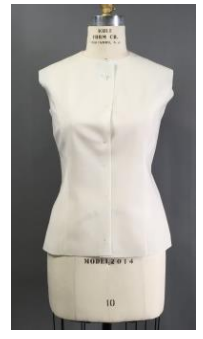

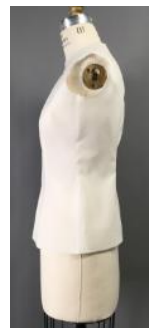

(a) JK3

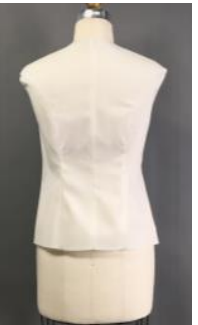

Figure 10. Jacket bodices for Body 3

As shown in Figure 8, JK1 looks better and has fewer wrinkles in the upper bust, armpit and back when compared with JK1N. Without taking into account the vertical proportions, the position of the jacket's bust line does not correspond to the body's bust line. The bust line of $\mathrm{JK} 1 \mathrm{~N}$ is higher than that of Body 1 . Thus, the upper parts of the bust line have wrinkles owing to the large amount of ease, and the lower part of the bust line is stretched and has wrinkles. In the case of Body 2, JK2 looks better than JK2N. Similar to the case for Body 1, there are wrinkles in the upper parts of the bust line. Constriction of the side line of JK2 is similar to that of the Standard Jacket bodice in Figure 4. However, the constriction of JK2N is angular and not similar to that of the Standard Jacket bodice. This is due to the difference in the waist line between the jacket and body. The results for JK3 and JK3N are similar to those of JK2 and JK2N. It is thus necessary to realize better fitting and fewer wrinkles using the multiplication factors for the horizontal dimension and adjustment for the vertical proportion.

Table 3 gives the measurements and ease allowance of the bust line, waist line and hip line for target bodies and jackets with and without adjusting vertical proportions. The ease amount of the waist for JK1N is greater than that of JK1 and the ease amount of the hip is less than that for JK1. The side line of JK1N is thus straighter than that of JK1. Likewise, JK2N has a straighter side line than JK2. In the case of JK3N, the ease amount of the bust is larger than that of JK3 even though the ease amounts of the waist and hip for JK3N are smaller than those for JK3. Therefore, the proportions of jackets without adjustment of the vertical proportions were unbalanced, resulting in a poorly fitting shape and wrinkles.

Table 3. Measurements and ease allowances of the bust line, waist line and hip line for three bodies and jackets with and without adjusting body vertical proportions

\begin{tabular}{|c|c|c|c|c|c|c|}
\hline Target body & Measurement & Body (cm) & $\begin{array}{c}\text { Jacket } \\
\text { name }\end{array}$ & $\begin{array}{c}\text { Jacket }(\mathrm{cm}) \\
\text { (percentage } \\
\text { ease) }\end{array}$ & $\begin{array}{c}\text { Jacket } \\
\text { name }\end{array}$ & $\begin{array}{c}\text { Jacket }(\mathrm{cm}) \\
\text { (percentage } \\
\text { ease) }\end{array}$ \\
\hline \multirow{3}{*}{$\begin{array}{l}\text { Standard } \\
\text { Body }\end{array}$} & Bust & 87.0 & \multirow{3}{*}{$\begin{array}{c}\text { Standard } \\
\text { Jacket } \\
\text { bodice }\end{array}$} & $94.3(8.4 \%)$ & \multirow[t]{3}{*}{ Y } & \\
\hline & Waist & 63.0 & & $80.9(28.4 \%)$ & & \\
\hline & Hip & 93.0 & & $103.1(10.9 \%)$ & & \\
\hline \multirow{3}{*}{ Body 1} & Bust & 87.0 & \multirow{3}{*}{ JK1 } & $94.9(9.1 \%)$ & \multirow{3}{*}{ JK1N } & $95.5(9.8 \%)$ \\
\hline & Waist & 67.0 & & $83.9(28.4 \%)$ & & $86.6(29.2 \%)$ \\
\hline & Hip & 94.0 & & $104.7(11.4 \%)$ & & $102.5(9.0 \%)$ \\
\hline \multirow{3}{*}{ Body 2} & Bust & 82.0 & \multirow{3}{*}{ JK2 } & $95.1(9.7 \%)$ & \multirow{3}{*}{$\mathrm{JK} 2 \mathrm{~N}$} & $87.8(7.1 \%)$ \\
\hline & Waist & 59.0 & & $74.5(26.3 \%)$ & & $76.6(29.8 \%)$ \\
\hline & Hip & 87.0 & & $100.4(15.4 \%)$ & & $98.6(13.3 \%)$ \\
\hline \multirow{3}{*}{ Body 3} & Bust & 90.2 & \multirow{3}{*}{ JK3 } & $98.0(8.6 \%)$ & \multirow{3}{*}{ JK3N } & $100.3(11.2 \%)$ \\
\hline & Waist & 66.0 & & $82.9(25.6 \%)$ & & $81.0(22.7 \%)$ \\
\hline & Hip & 94.0 & & $105.2(11.9 \%)$ & & $102.0(8.5 \%)$ \\
\hline
\end{tabular}


To show these differences graphically, cross-sectional shapes of jackets in the upper and lower parts of the bust line were compared. We measured the cross-sectional shapes $5 \mathrm{~cm}$ below and 5 $\mathrm{cm}$ above the bust line; we refer to these cross-sections as the under-bust and upper-bust crosssections respectively. The shapes are compared in Figures 11-13. Under-bust and upper-bust cross-sections of JK1N, JK2N and JK3N show more undulation than those of JK1, JK2 and JK3. The undulations correspond to wrinkles of JK1N, JK2N and JK3N in Figures 8-10.

The measurements and ease allowance for target bodies and jackets are given in Table 4. Under-bust lines of JK1N, JK2N and JK3N have less ease than those of JK1, JK2 and JK3. This is because the distance of NP-BL is shorter for the Standard Body $(13 \mathrm{~cm})$ than for the other bodies $(15-17 \mathrm{~cm})$ as shown in Table 1. Under-bust parts of JK1N, JK2N and JK3N have less ease for forming a smooth surface under the bust. These parts were thus pulled. Wrinkling then occurred and produced the undulating lines in Figures 11-13. Meanwhile, upper-bust lines of $\mathrm{JK} 1 \mathrm{~N}, \mathrm{JK} 2 \mathrm{~N}$ and JK3N had more ease than JK1, JK2 and JK3, which also caused the undulating lines. These results show that the jackets made without adjusting the vertical body proportions do not fit bodies having different proportions.

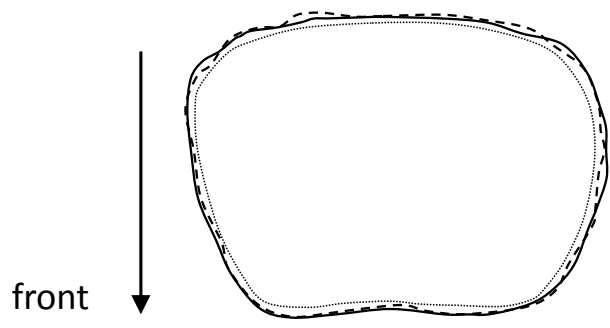

(a) Under-bust

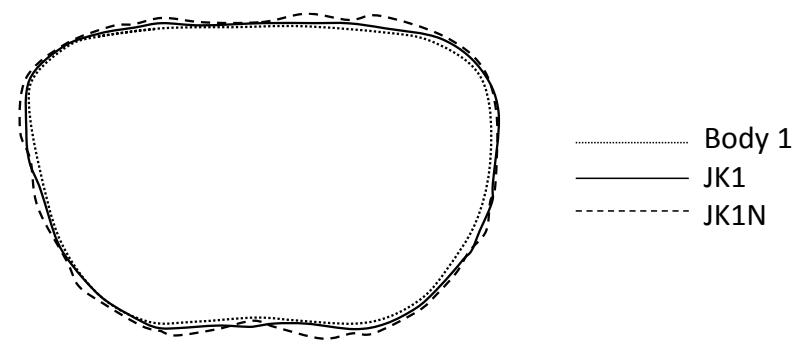

(b) Upper-bust

Figure 11. Comparisons of cross-sections for Body 1, JK1 and JK1N

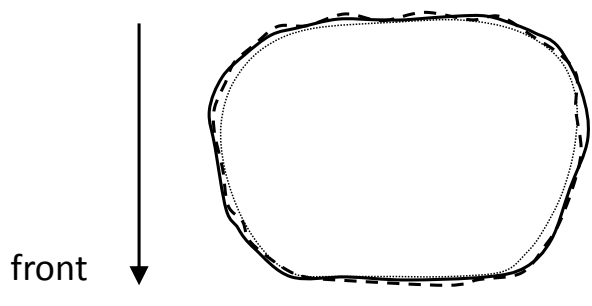

(a) Under-bust

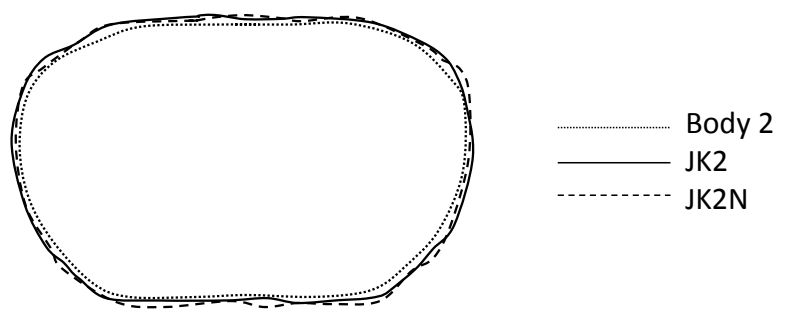

(b) Upper-bust

Figure 12. Comparisons of cross-sections for Body 2, JK2 and JK2N

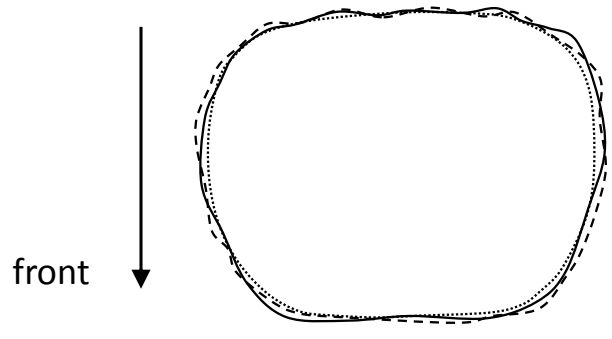

(a) Under-bust

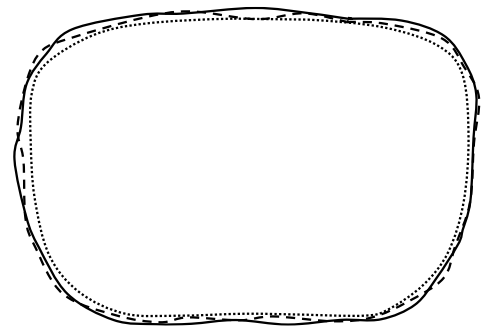

(b) Upper-bust

Figure 13. Comparisons of cross-sections for Body 3, JK3 and JK3N 
Table 4. Measurements and ease allowance of under the bust and the upper bust for three bodies and jackets

\begin{tabular}{|c|c|c|c|c|c|c|}
\hline $\begin{array}{l}\text { Target } \\
\text { body }\end{array}$ & Measurement & $\begin{array}{l}\text { Body } \\
(\mathrm{cm})\end{array}$ & $\begin{array}{l}\text { Jacket } \\
\text { name }\end{array}$ & $\begin{array}{c}\text { Jacket }(\mathrm{cm}) \\
\text { (percentage } \\
\text { ease) }\end{array}$ & $\begin{array}{l}\text { Jacket } \\
\text { name }\end{array}$ & $\begin{array}{c}\text { Jacket }(\mathrm{cm}) \\
\text { (percentage } \\
\text { ease) }\end{array}$ \\
\hline \multirow{2}{*}{$\begin{array}{l}\text { Standard } \\
\text { Body }\end{array}$} & Upper bust & 90.5 & \multirow{2}{*}{$\begin{array}{c}\text { Standard } \\
\text { Jacket } \\
\text { bodice }\end{array}$} & $95.1(5.1 \%)$ & \multirow{2}{*}{\multicolumn{2}{|c|}{$\mathrm{X}^{2}$}} \\
\hline & Under bust & 74.1 & & $84.9(14.6 \%)$ & & \\
\hline \multirow{2}{*}{ Body 1} & Upper bust & 88.1 & \multirow{2}{*}{ JK1 } & $92.6(5.1 \%)$ & \multirow{2}{*}{ JK1N } & $95.0(7.8 \%)$ \\
\hline & Under bust & 73.5 & & $84.4(14.8 \%)$ & & $82.8(12.6 \%)$ \\
\hline \multirow{2}{*}{ Body 2} & Upper bust & 88.2 & \multirow{2}{*}{$\mathrm{JK} 2$} & $92.8(5.2 \%)$ & \multirow{2}{*}{ JK2N } & $96.1(9.0 \%)$ \\
\hline & Under bust & 71.9 & & $82.0(14.0 \%)$ & & $81.3(13.1 \%)$ \\
\hline \multirow{2}{*}{ Body 3} & Upper bust & 92.5 & \multirow{2}{*}{ JK3 } & $97.0(4.9 \%)$ & \multirow{2}{*}{$\mathrm{JK} 3 \mathrm{~N}$} & $100.3(8.43 \%)$ \\
\hline & Under bust & 78.5 & & $90.3(15.0 \%)$ & & $88.7(13.0 \%)$ \\
\hline
\end{tabular}

We therefore conclude that, in 3D garment modeling and pattern making, not only the horizontal dimensions but also the body vertical proportions should be taken into account to make appropriate patterns for bodies of different sizes and proportions. The proposed method can therefore be used as a method of changing size, especially in creating individualized garments or garments for different targets.

\section{Conclusions}

In this study, a new method of upper-body garment modeling was proposed to perform 3D pattern making for different body sizes and vertical proportions. So that a garment model fits a target body in terms of both the girth and vertical proportion, the target body is expanded horizontally and then expanded or contracted vertically using preliminarily obtained horizontal multiplication factors and a vertical proportion adjustment.

To verify the validity of the proposed method, jacket models for three bodies of different sizes and proportions were made. By developing the models, bodice patterns were obtained and bodices of jackets were sewn. In addition, to confirm the effect of adjusting vertical proportions, these bodices of jackets were compared with those obtained without adjusting vertical proportions.

With the proposed method, jacket bodices were successfully made and were fitted on the target bodies while preserving the original shape. Jackets bodices made without taking into account the vertical proportions had many wrinkles and shapes that were deformed owing to the different vertical proportions. The poor fit was also seen in cross-sections around the bust line.

The results indicate that the proposed method is effective in 3D garment modeling for different sizes and vertical proportions of bodies. The body vertical proportion is a factor that cannot be neglected in 3D garment modeling.

The proposed method is applicable to size changes and grading when making individually tailored garments or ready-to-wear garments for different targets.

\section{Acknowledgements}

This work was supported by JSPS (Japan Society for the Promotion of Science) KAKENHI under grant numbers 24220012. 


\section{References}

Apeagyei, P.R. (2010), Application of 3D body scanning technology to human measurement for clothing Fit. International Journal of Digital Content Technology and its Applications, Vol. 4, No. 7, pp. 58-68.

Lim, H. and Istook, C. L. (2012), "Automatic pattern generation process for made-tomeasure", Journal of Textile and Apparel, Technology and Management, Vol. 7, No. 4, pp. $1-11$.

Bye, E., LaBat, K., McKinney, E. and Kim, D.E. (2008), "Optimized pattern grading", International Journal of Clothing Science and Technology, Vol. 20, No. 2, pp. 79-92.

Cichocka, A., Bruniaux, P. and Frydrych, I. (2014). "3D Garment Modelling-Creation of a Virtual Mannequin of the Human Body", Fibres \& Textiles in Eastern Europe, Vol. 22, No. 6(108), pp. 123-131.

Cooklin, G. (1991), Pattern Grading for Women's Clothing, Wiley-Blackwell, Oxford, pp. 40-43.

Cho, Y.S., Komatsu, T., Inui, S., Takatera, M., Shimizu, Y. and Park, H. (2006a), "Individual pattern making using computerized draping method for clothing", Textile Research Journal, Vol. 76, No. 8, pp. 646-654.

Cho, Y.S., Komatsu, T., Takatera, M., Inui, S., Shimizu, Y. and Park, H. (2006b), "Posture and depth adjustable 3D body model for individual pattern making", International Journal of Clothing Science and Technology, Vol. 18, No. 2, pp. 96-107.

Cho, Y.S., Okada, N., Park, H.J., Takatera, M., Inui, S. and Shimizu, Y. (2005), "An interactive body model for individual pattern making", International Journal of Clothing Science and Technology, Vol. 17, No. 2, pp. 91-99.

Heisey, F., Brown, P. and Johnson, R.F. (1990), "Three-dimensional pattern drafting Part II: Garment modeling”, Textile Research Journal, Vol. 60, No. 12, pp. 731-737.

Hsiao, S.W. and Chen, R.Q. (2013), "A study of surface reconstruction for 3D mannequins based on feature curves", Computer-Aided Design, Vol. 45, No. (11), pp. 1426-1441.

Hyo Kim, C., Hwan Sul, I., Kyu Park, C. and Kim, S. (2010), "Automatic basic garment pattern generation using three-dimensional measurements", International Journal of Clothing Science and Technology, Vol. 22, No. 2/3, pp. 101-113.

Imaoka H., Shibuya A. and Aisaka N. (1989), "Automatic paper pattern making using mechanical development method of a curved surface on a plane surface", Sen'i Gakkaishi, Vol. 45, No. 10, pp. 427-434.

Kim, S. (2015), "Analysis of human body surface shape using parametric design method", International Journal of Clothing Science and Technology, Vol. 27, No. 3, pp. 434-446.

Price, J. and Zamkoff, B. (1996), Grading Techniques for Fashion Design, second edition, Fairchild Publications, USA, pp. 2-14.

Sul I.H., Kang T.J. (2006). "Interactive garment pattern design using virtual scissoring method", International Journal of Clothing Science and Technology, Vol. 18, No. 1, pp. 31-42.

Takatera, M. and Kim, K.O. (2013), "Improvement of individualized pattern making using surface flattening technique", Journal of Fiber Bioengineering and informatics, Vol. 6, No. 4, pp. 467-480.

Umetani, N., Kaufman, D.M., Igarashi, T. and Grinspun, E. (2011), "Sensitive couture for interactive garment modeling and editing”, ACM Transactions on Graphics, Vol. 30, No. 4, pp. 90.

Wang, Y. and Huang, H. (2011), "Three Dimensional Pattern Grading Based on Deformable Body Features and 3D Developable Surface", Journal of Fiber Bioengineering and Informatics, Vol. 4, No. 2, pp. 115-128.

Yang, Y.C. and Zhang. W.Y. (2007), "Prototype garment pattern flattening based on individual 3D virtual dummy", International Journal of Clothing Science and Technology, Vol. 19, No. 5, pp. 334-348. 
Zhang, J., Innami, N., Kim, K.O. and Takatera, M. (2015), "Upper Garment 3D Modeling for Pattern Making”, International Journal of Clothing Science and Technology, Vol. 27, No.6, pp.852-869.

Zhang M., Kamijo M., Takatera M., Furukawa T. and Shimizu Y. (2000), "An interactive pattern making and 3D simulation system of collars", Kansei Engineering International, Vol. 1, No. 4, pp. 1-10. 